\title{
ICAM-3 enhances the migratory and invasive potential of human non-small cell lung cancer cells by inducing MMP-2 and MMP-9 via Akt and CREB
}

\author{
JONG KUK PARK ${ }^{1}$, SEON HO PARK ${ }^{1}$, KWANGSUP SO ${ }^{1}$, IN HWA BAE ${ }^{1}$, \\ YOUNG DO YOO ${ }^{2}$ and HONG-DUCK UM ${ }^{1}$ \\ ${ }^{1}$ Laboratory of Radiation Tumor Physiology, Korea Institute of Radiological and Medical Sciences, \\ 215-4 Gongneung-Dong, Nowon-Gu, Seoul 139-706; ${ }^{2}$ Graduate School of Medicine, \\ Korea University, 126-1 5ka, Anam-Dong, Sungbuk-Gu, Seoul 136-705, Korea
}

Received August 10, 2009; Accepted September 25, 2009

DOI: 10.3892/ijo_00000489

\begin{abstract}
We have previously reported that intercellular adhesion molecule-3 (ICAM-3) is associated with an increase of cellular radio-resistance and cancer cell proliferation. In this study, we hypothesized that ICAM-3 has an additional effect on cancer cell migration and invasion because molecules induced by ICAM-3 are known as regulators of cell migration and invasion. To examine this hypothesis, we used NCI-H1299 non-small cell lung cancer (NSCLC) cell line (p53 and PTEN null cell) and constructed an ICAM-3over-expressing stable transfectant, which exhibited increased cell migration and invasion. The increased migration and invasion resulted from up-regulation of expression and activities of MMP-2 and MMP-9. ICAM-3 also increased Akt phosphorylation, which caused an increase in cellular migration/invasion and MMP activities. Activity of several transcriptional factors located downstream in the Akt pathway was also tested, and constitutive activation of adenosine 3', 5'-monophosphate response element-binding protein (CREB) by ICAM-3 was detected. Blockage of the Akt pathway attenuated CREB activation, and a decrease in CREB expression reduced cellular migration/invasion and activity of MMPs. This result indicates that CREB functions in the signaling pathway between Akt and MMP. We also showed ICAM-3-induced cell migration and invasion in NCIH460 NSCLC cells (wild-type $p 53$ and PTEN cell) through the same signaling pathway. Taken together, our findings suggest that ICAM-3 stimulates cancer cell migration/ invasion via ICAM-3/Akt/CREB/MMP pathway regardless of p53 and PTEN status, and this reflects the possibility that
\end{abstract}

Correspondence to: Dr Jong Kuk Park or Dr Hong-Duck Um, Laboratory of Radiation Tumor Physiology, Korea Institute of Radiological and Medical Sciences, 215-4 Gongneung-Dong, Nowon-Gu, Seoul 139-706, Korea

E-mail: jkpark@kcch.re.kr; hdum@kcch.re.kr

Key words: ICAM-3, migration, invasion, Akt, CREB, MMP
ICAM-3 could be considered as a candidate for anti-cancer drug development and as a cancer diagnostic marker.

\section{Introduction}

Cancer cells are characterized by various activities that are distinct from those of normal cells. Invasion and metastasis are major characteristics of cancer, composed of multi-step processes that are critically dependent on the ability of cells to move away from original cancer site, which involves gaining access to the vasculature of the lymphatic system, to move from the primary tumor to distant sites where they grow in a favorable microenvironment at a secondary location (1-3). Therefore, migration of cancer cells and degradation of the extracellular matrix (ECM) are important steps in cancer invasion and metastasis. Expression or activities of various ECM-degrading enzymes, such as matrix metalloproteinase (MMP) family proteins, are required and various intracellular signaling pathways involving phosphoinositide 3-kinase (PI3 kinase)/Akt and mitogen-activated protein kinase (MAPK) signaling pathways induce ECM-degrading enzyme activity (1-3). Because most of these intracellular signaling pathways inducing invasion and metastasis commence at the cell surface $(4,5)$, cell surface molecules play an important role in cell invasion and metastasis. Neural cell adhesion molecule (NCAM) and carcinoembryonic antigen (CEA) are wellknown cell surface molecules that are involved in invasion and metastasis of multiple myeloma and colorectal cancers $(6,7)$.

The ICAM family proteins comprises cell surface molecules that are homologous to NCAM and are members of the single passed type 1 immunoglobulin superfamily (IgSF) that are anchored at the cellular membrane (8). The ICAM family consists of five subfamilies (ICAM-1 to ICAM-5) of heavily glycosylated cell surface receptors with common functional or structural homologies (9-12). These proteins contain extracellular domains, transmembrane residues and cytoplasmic tail residues. The extracellular domains of ICAM protein have roles in the immune response and inflammation through various cell-cell interactions. ICAM-3 binds to the 
leukocyte function-associated antigens (LFAs) and Mac-1, which in turn induces a T cell response (13). ICAM-3 is also involved in dendritic cell functions by binding to C-type lectin dendritic cell-specific ICAM-grabbing non-integrin (DC-SIGN) $(9,14)$. Interestingly, the ICAM proteins appear to have a dual role in cancer. ICAM molecules may target and block tumor progression by stimulation of an immune response such as leukocyte activation $(15,16)$. Conversely, other investigations have shown that ICAM molecules are involved in cancer malignancy because their increased expressions are associated with a poor diagnosis, lower survival rates and metastasis in some cancers including melanoma, breast cancer and leukemia (17-24). We have also reported that an increase of ICAM-3 expression in several cancer cells and specimens of cervical cancer patients induced enhanced radio-resistance by the activation of focal adhesion kinase (FAK) (25) and promoted cancer cell proliferation by the activation of Akt and p44/42 MAPK (26). Therefore, these previous reports imply that ICAM-3 has various undefined roles in cancer.

In this study, we investigated whether ICAM-3 increased cell migration and invasion because it has been shown that ICAM family proteins activate several proteins such as integrin molecules and IKK proteins involved in cancer invasion $(15,17,18)$. As ICAM-3 over-expressing stable transfectant displayed elevated migration and invasion ability, we attempted to identify the molecule driving migration and invasion. MMP proteins showed increased expressions and activities, which resulted in increased cell migration and cancer invasion in ICAM-3 stable transfectants. To investigate the intracellular signaling between ICAM-3 and MMP proteins, we tried to detect components involved in ICAM-3induced cell migration and invasion. Because PI3 kinase/Akt pathway and p44/42 MAPK are located downstream in the ICAM-3 pathway, these molecules and those downstream from them were investigated preferentially, and we found that CREB, one of the transcription factors, is also involved in cell migration and invasion activated by ICAM-3 signaling. Therefore, these results provided us with more information regarding ICAM-3-related signaling pathway and defined the role of ICAM-3 in invasion and metastasis of cancer cells.

\section{Materials and methods}

Cell culture and reagents. The human NSCLC cell lines NCIH460 and NCI-H1299 were purchased from the American Type Culture Collection (Rockville, MD, USA). NCI-H1299 cells were grown in RPMI-1640 (Gibco-Invitrogen, Grand Island, NY, USA) medium supplemented with $10 \%$ fetal bovine serum (Gibco-Invitrogen) and 1\% penicillin-streptomycin (Invitrogen) under a humidified atmosphere with 5\% $\mathrm{CO}_{2}$ at $37^{\circ} \mathrm{C}$. LY294002, OA-Hy and MMP-9 inhibitor I were purchased from Calbiochem (La Jolla, CA, USA).

To determine the function of ICAM-3, we transfected NCI-H1299 cells with pcDNA3 and wild-type ICAM-3 (pcDNA3-ICAM-3) plasmids using Lipofectamine 2000 (Invitrogen, Carlsbad, CA, USA). Colony selection and maintenance of stably transfected cells were performed with the use of G418 (Invitrogen). In siRNA treatment, NCI-H1299 stable transfectant cells were transiently transfected with a control siRNA (sc-37007; Santa Cruz Biotechnology, Santa Cruz, CA, USA) and $C R E B$ siRNA (Santa Cruz Biotechnology, sc-29281) using Lipofectamine 2000 (Invitrogen), according to the protocol recommended by the manufacturer. NCIH460 and NCI-H1299 cells were transfected transiently with pcDNA3 and wild-type ICAM-3 (pcDNA3-ICAM-3) plasmids using Lipofectamine 2000 (Invitrogen).

Immunoblot assay. Cells were harvested and washed twice with ice-cold PBS. Cells were dissolved with $50 \mu 1$ of RIPA buffer [150 mM NaCl, $1 \%$ NP-40, 0.5\% deoxycholic acid, $0.1 \%$ SDS, $50 \mathrm{mM}$ Tris ( $\mathrm{pH} 8.0)$ ] containing phosphatase and protease inhibitors for $30 \mathrm{~min}$ at $4^{\circ} \mathrm{C}$. Lysates were separated using 12\% SDS-PAGE (sodium dodecyl sulfatepolyacrylamide gel electrophoresis) and proteins were transferred to a nitrocellulose membrane (Invitrogen). Membranes were incubated with primary antibodies according to the manufacturer's protocol, subsequently washed with PBS-T (phosphate-uffered saline Tween-20), and were incubated with an appropriate secondary antibody dependent to primary antibody. An ECL detection system (GE Healthcare UK, Chalfont St. Giles, UK) was used to detect the target proteins. Anti-ICAM-3 antibody was obtained from Abcam (Cambridge, UK), while phospho-Akt, Akt and phospho-CREB, CREB antibodies were obtained from Cell Signaling Technology (Danvers, MA, USA). MMP-2 and MMP-9 antibodies were purchased from Calbiochem (La Jolla).

RNA isolation, cDNA synthesis and PCR. Total RNA isolation for reverse transcription (RT)-PCR was performed with TRIzol (Invitrogen) according to the manufacturer's protocol and complementary DNA was synthesized with $5 \mu \mathrm{l}$ of StrataScript buffer (10X), $1 \mu 1$ of ribonuclease inhibitor (40 U/ $\mu 1), 2 \mu \mathrm{l}$ of dNTP mixture $(100 \mathrm{mM})$ and $1 \mu \mathrm{l}$ of StrataScript reverse transcriptase $(50 \mathrm{U} / \mu \mathrm{l})$. The reaction was performed for $1 \mathrm{~h}$ at $42^{\circ} \mathrm{C}$. Reagents were obtained from Stratagene (La Jolla).

The concentration of each synthesized cDNA was determined. PCR reactions were performed with the following primers: ICAM-3, 5'-GTGAACTGCAGTACTGATTGT-3' and 5'-GGTGATGTTAGAGGAGCCTGT-3'; MMP-2, 5'CAGGCTCTTCTCCTTTCACAAC-3' and 5'-AAGCCACG GCTTGGTTTTCCTC-3'; MMP-9, 5'-TGGGCTACGTGAC CTATGACAT-3' and GCCCAGCCCACCTCCACTCCTC-3'; B2-microglobulin (B2M), 5'-GTGGAGCATTCAGACTTGT CTTTCAGCA-3' and 5'-TTCACTCAATCCAAATGCG GCATCTTC-3'.

Wound healing assay. To perform the wound healing assay, stably transfected cells were seeded $\left(3 \times 10^{5}\right)$ on $35-\mathrm{mm}$ cell culture dishes and were incubated overnight under a humidified atmosphere with $5 \% \mathrm{CO}_{2}$ at $37^{\circ} \mathrm{C}$. The wound was generated with the tip of a micropipette after confirming the full confluence of the seeded cells, and cells were washed twice with serum-free medium. Serum-free medium containing $0.1 \%$ BSA was added, and wound healing was observed under a microscope after $24 \mathrm{~h}$.

Gelatin zymography. PcDNA3 and ICAM-3 NCI-H1299 stable transfectants were seeded on a 6-well plate, washed 
with serum-free medium and incubated for $24 \mathrm{~h}$ in serumfree medium under a humidified atmosphere with $5 \% \mathrm{CO}_{2}$ at $37^{\circ} \mathrm{C}$. Incubated serum-free media were loaded and proteins were separated with the use of a $0.1 \%$ gelatin SDS-PAGE gel $(10 \%)$. After separation, gel renaturation was performed with $2.5 \%$ Triton $\mathrm{X}-100$ buffer for $1 \mathrm{~h}$, and the gelatin gel was incubated with developing solution $(50 \mathrm{mM}$ Tris $\mathrm{pH} 7.5$, $20 \mathrm{mM} \mathrm{NaCl}, 10 \mathrm{mM} \mathrm{CaCl}_{2}, 1 \mu \mathrm{M} \mathrm{ZnCl}_{2}, 0.1 \% \mathrm{NaN}_{3}$ ) for $1 \mathrm{~h}$. The developing buffer was changed after primary incubation, and a secondary incubation was performed for $24 \mathrm{~h}$ at $37^{\circ} \mathrm{C}$. The gel was stained with 5\% Coomassie Brilliant Blue stain (Sigma, St. Louis, MO, USA).

Migration and invasion assay. Migration and invasion assays were performed as described by Bae et al (27). Collagen (3 $\mu \mathrm{g} / \mu \mathrm{l}$, Sigma)-coated transwells $(0.8 \mu \mathrm{m}$ pore; Corning, NY, USA) were used to detect cell migration and Matrigelcoated transwells $(1 \mathrm{mg} / \mathrm{ml}$, Invitrogen) were used to detect cell invasion. Cells $\left(2 \times 10^{4}\right)$ were washed with serum-free media twice and then added to the upper chamber of each transwell. Serum-free medium containing $0.1 \%$ BSA was added to the lower chamber, and the transwells were incubated for $18 \mathrm{~h}$ with $5 \% \mathrm{CO}_{2}$ at $37^{\circ} \mathrm{C}$. Cell staining was performed with deep quick solution (Merck, Whitehouse Station, NJ, USA), according to the manufacturer's protocol. Stained cells were counted under a microscope and statistical analysis was performed.

Electrophoretic mobility shift assay. PcDNA3 and ICAM-3 NCI-H1299 stable transfectants were harvested and nuclei were isolated as previously described (28). Electrophoretic mobility shift assay (EMSA) system was purchased from Promega (Madison, WI, USA) and all procedures were performed as described by the manufacturer.

Statistical analysis. All experiments were repeated at least three times. Data were calculated with the use of GraphPad Prism software (GraphPad Software, La Jolla), and differences between experimental groups were determined with use of the t-test. Error bars indicate standard deviations (SD) and p-values were calculated.

\section{Results}

ICAM-3 enhances migration and invasion. To investigate the role of ICAM-3 in cell migration and invasion, we made ICAM-3 over-expressing stable tansfectants of NCI-H1299 cells (NCI-H1299 ICAM-3 stable transfectant), as described in Materials and methods. NCI-H1299 cells transfected with the pcDNA3 vector (NCI-H1299 pcDNA3 stable transfectant) served as the control. High expression of ICAM-3 in NCIH1299 ICAM-3 stable transfectants was confirmed by RTPCR (Fig. 1A, upper panel) and by immunoblot assay (Fig. 1A, lower panel). We observed high-level expression of exogenous ICAM-3 in NCI-H1299 ICAM-3 stable transfectants, whereas there was no detectable expression of ICAM-3 in NCI-H1299 pcDNA3 stable transfectants. This result indicates that NCI-H1299 cells did not express ICAM-3 to detectable level in the transcriptional or translational step. Cell migration was analyzed by wound-healing assay using
0.1\% BSA-containing serum-free media (Fig. 1B, upper panel). Wounds generated on the cell plate of NCI-H1299 ICAM-3 stable transfectants were almost completely healed at $24 \mathrm{~h}$, but wounds of NCI-H1299 pcDNA3 stable transfectants were not. Enhanced migration by NCI-H1299 ICAM-3 stable transfectants was confirmed by quantitative analysis using collagen-coated transwells (Fig. 1B, lower panel). The mean migrated proportion of NCI-H1299 pcDNA3- and ICAM-3 stable transfectants were about 100 and $168.8 \%$, respectively. Enhanced invasion of NCI-H1299 ICAM-3 stable transfectants was confirmed by the use of Matrigelcoated transwells (Fig. 1C). The mean invaded proportion of pcDNA3- and ICAM-3 stable transfectant cells was approximately 100 and $176.1 \%$, respectively. The results indicate that ICAM-3 enhanced cellular migration and invasion in this system.

Enhanced migration and invasion by ICAM-3 is mediated by $M M P-2$ and MMP-9. To further elucidate the mechanism underlying ICAM-3-induced migration and invasion, we determined MMP-2 and MMP-9 expression levels (Fig. 2A). The NCI-H1299 ICAM-3 stable transfectant cells showed a two-fold increase in expression of MMP-2 and MMP-9 compared with the control cells. Based on this finding, we conclude that ICAM-3 increased the expression of both MMP-2 and MMP-9. Gelatin zymography was performed to assess MMP-2 and MMP-9 activity (Fig. 2B). As expected, NCI-H1299 ICAM-3 stable transfectants showed elevated MMP-2 and MMP-9 activities. The next step was to determine if increased MMP-2 and MMP-9 expression or activity induced by ICAM-3 directly affect cell migration (Fig. 2C) or invasion (Fig. 2D). We used specific pharmacological inhibitors of the MMP proteins. Cells were treated with $10 \mu \mathrm{M}$ OA-Hy (an MMP-2-specific inhibitor), $500 \mathrm{ng} / \mu 1 \mathrm{MMP}-9$ inhibitor I (an MMP-9-specific inhibitor) or a combination of OA-Hy and MMP-9 inhibitor I, and migration and invasion assays were performed. In both assays, migration and invasion rates of pcDNA3 and ICAM-3 stable transfectant cells treated with the individual inhibitors were reduced by approximately $20-30 \%$, whereas the combination of both inhibitors reduced migration and invasion rates by about $40-80 \%$, as compared to controls. These results suggested that both MMP-2 and MMP-9 activations induced by ICAM-3 directly participate in increased migration and invasion.

Akt is involved in ICAM-3-induced cell migration and invasion. As our previous studies have shown that ICAM-3 induces activation of Akt or the p44/42 MAPK pathway, we attempted to clarify if Akt or p44/42 MAPK contributes to cellular migration and invasion in the present system. First, we performed an immunoblot assay for phospho-Akt (Fig. 3A) and employed gelatin zymography using conditioned mediatreated with DMSO or LY294002 (20 $\mu \mathrm{M}$; a PI3 kinase/ Akt pathway inhibitor) (Fig. 3B). We detected an increase of the phospho-Akt level in ICAM-3 stable transfectant cells (Fig. 3A) and a decrease in MMP-2 and MMP-9 activity following LY294002 treatment (Fig. 3B). These results suggested that Akt is involved in the signaling induced by ICAM-3 resulting in increased MMP-2 or MMP-9 activity induced by ICAM-3. As modification of MMP-2 and MMP-9 
A
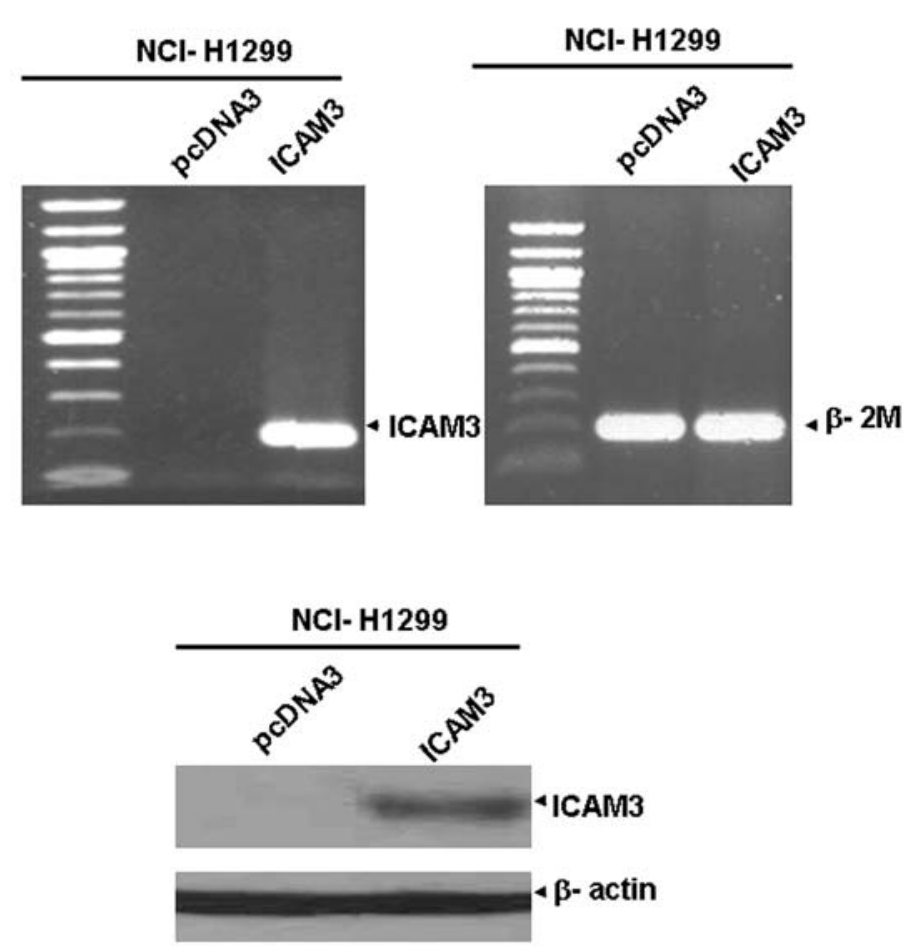

B
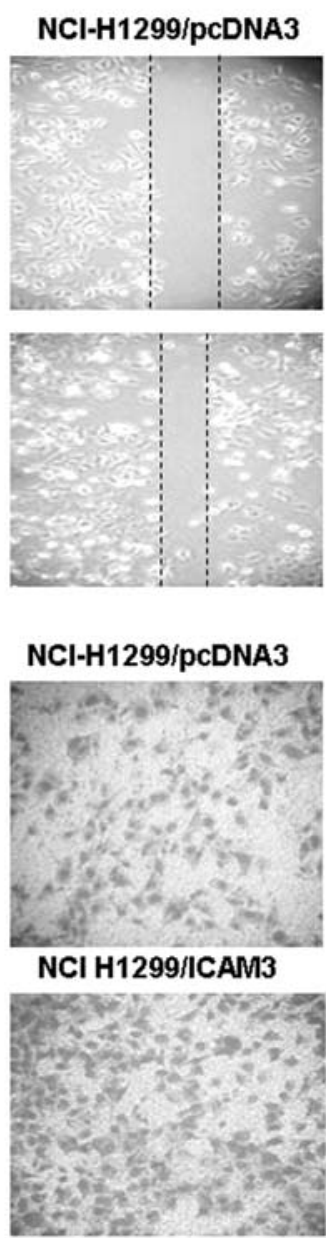

NCI H1299/CAM3

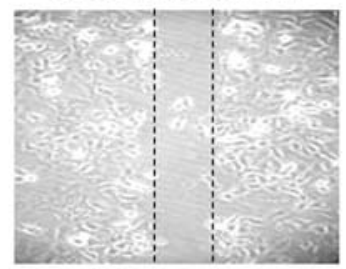

0 hrs

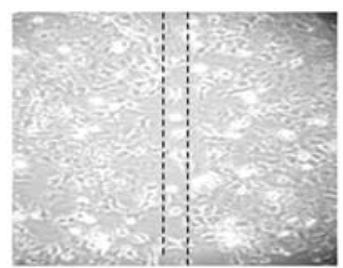

$24 \mathrm{hrs}$

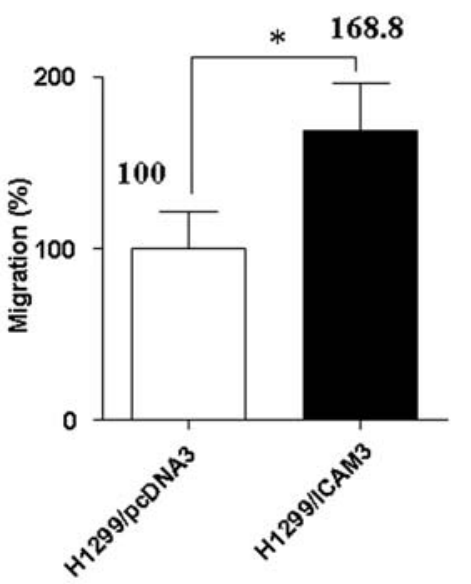

C

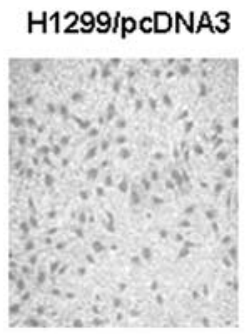

H1299IICAM3
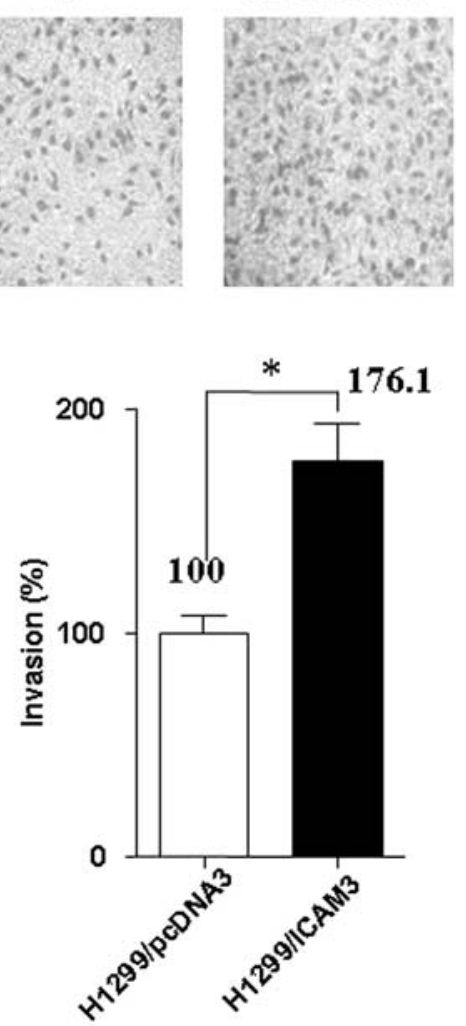

Figure 1. ICAM-3 increases migration and invasion. (A) Construction and confirmation of a control mock vector (pcDNA3) or ICAM-3 stable transfectants of NCI-H1299 cells. pcDNA3 and ICAM-3 represent NCI-H1299 cells transfected with pcDNA3 and ICAM-3, respectively. The upper panel depicts RT-PCR assay of stable transfectants. The molecular sizes of ICAM-3 and B-2M PCR products detected using RT-PCR were 186 base pairs and 190 base pairs, respectively. The lower panel shows an immunoblot assay of stable transfectants. ICAM-3 (110 kDa) and $\beta$-actin (43 kDa) expression was detected in stable transfectants. (B) NCI-H1299 ICAM-3 stable transfectant displayed increased cell migration. The upper panel shows the wound-healing assay, and the lower panel depicts migration assay using the transwell system. (C) NCI-H1299 ICAM-3 stable transfectants exhibited increased cell invasion. Migration data were calculated where each value represents the mean of three independent experiments, and error bars reflect standard deviations (SD). The p-values between NCI-H1299 pcDNA3 and ICAM3 stable transfectants were determined with use of the t-test. ${ }^{*} \mathrm{p}<0.05$.

activity affected cellular migration and invasion capacities, we investigated whether LY294002 might also affect these processes. In the migration and invasion assay, blocking of Akt with LY293002 inhibited cell migration and invasion (Fig. 3C and D). We therefore suggest that ICAM-3 activates Akt, which subsequently affects cellular migration and invasion through modulation of MMP-2 or MMP-9 activity. Interestingly, LY294002 also suppressed MMP-2 and MMP-9 activity in NCI-H1299 pcDNA3 stable transfectants as well as NCI-H1299 ICAM-3 stable transfectant. This phenomenon may be attributed to the capacity of LY294002 to inhibit the activity of Akt affected by other upstream signaling component in NCI-H1299 cells, especially because as this cell does not have the phosphatase and tensin homolog $(P T E N)$ gene, an endogenous intracellular inhibitor of PI3 
A

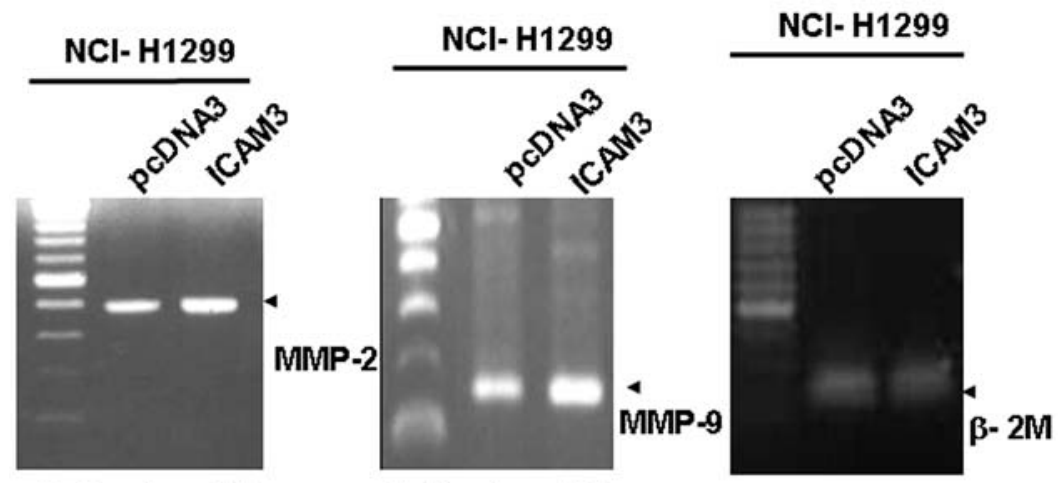

Ratio $1: 2.2$

Ratio $1: 2.6$

B

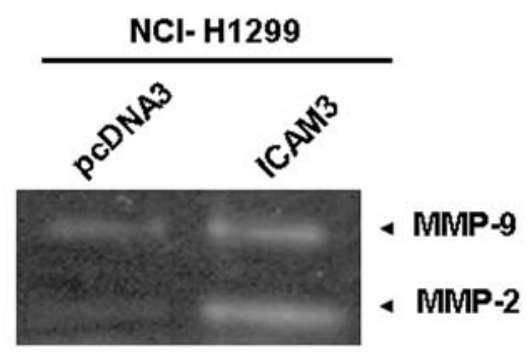

C

D
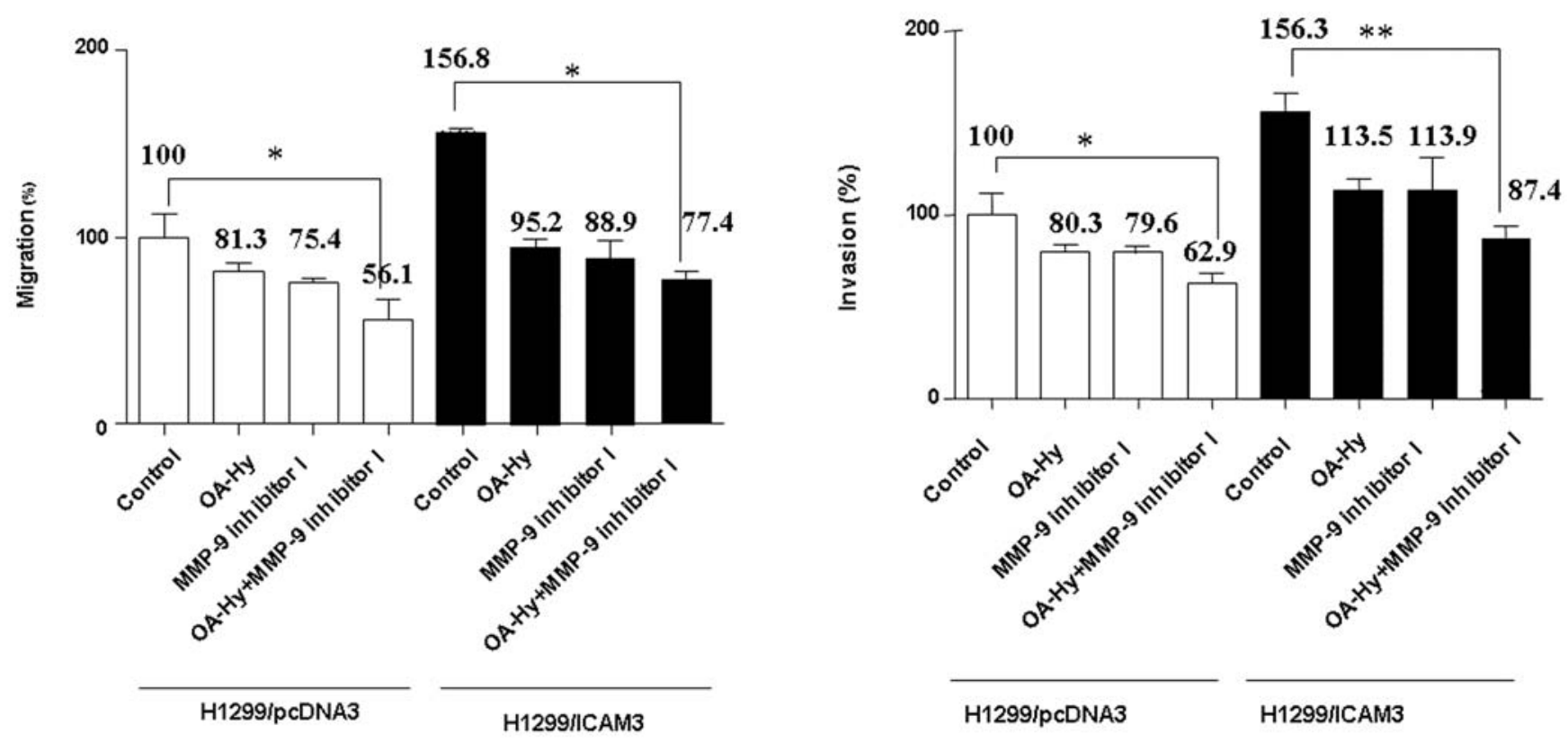

Figure 2. ICAM-3 enhances migration and invasion through activation of MMP-2 and MMP-9. (A) An increase in MMP-2 and MMP-9 expression was detected with RT-PCR. Relative expression ratios of MMP-2 and MMP-9 in NCI-H1299 pcDNA3 and ICAM-3 stable transfectants were detected and calculated with Gel Documentation system (Bio-Rad, USA). (B) Activity of MMP-2 or MMP-9 in NCI-H1299 pcDNA3- and ICAM-3 stable transfectants were detected using gelatin zymography. (C and D) Stable transfectants were pre-treated with specific inhibitors of MMP-2 (OA-Hy), MMP-9 inhibitor I, or a combination of OA-Hy and MMP-9 inhibitor I for $18 \mathrm{~h}$, and migration $(\mathrm{C})$ and invasion assays (D) were performed as described in Materials and methods. Numbers above each bar represent the mean of three independent experiments and error bars indicate standard deviations (SD). p-values were calculated using the t-test. ${ }^{*} \mathrm{p}<0.05 ;{ }^{* *} \mathrm{p}<0.001$. 
A

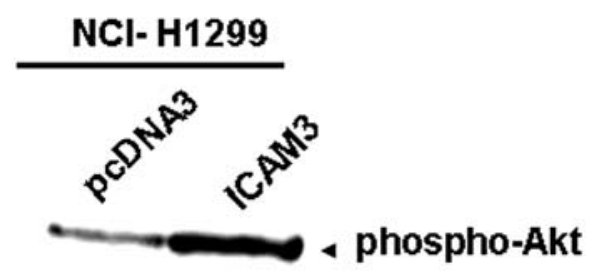

Akt

C

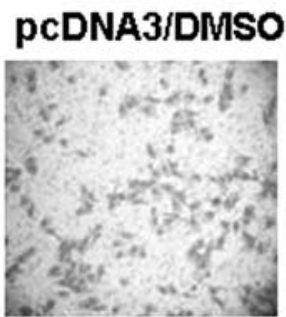

ICAM3/DMSO

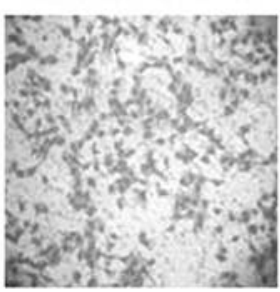

PCDNA3/LY

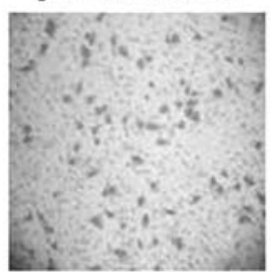

ICAM3LY

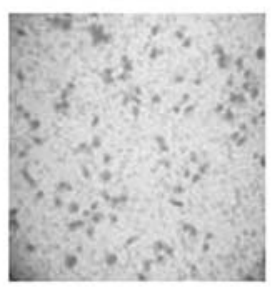

B

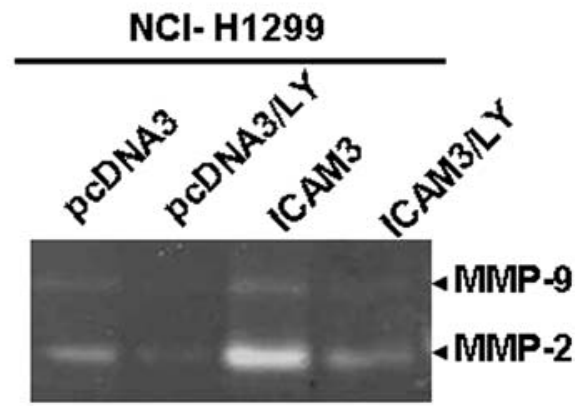

D

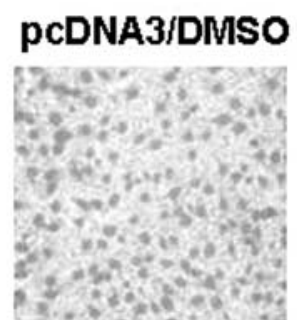

pCDNA3/Y

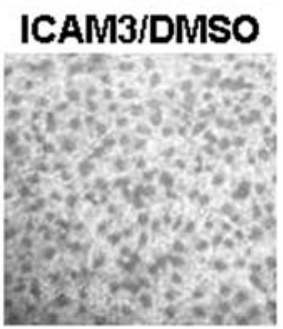

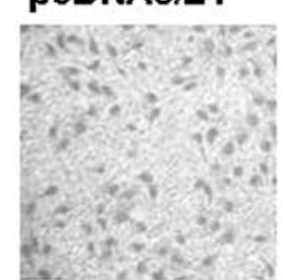

ICAM3LY
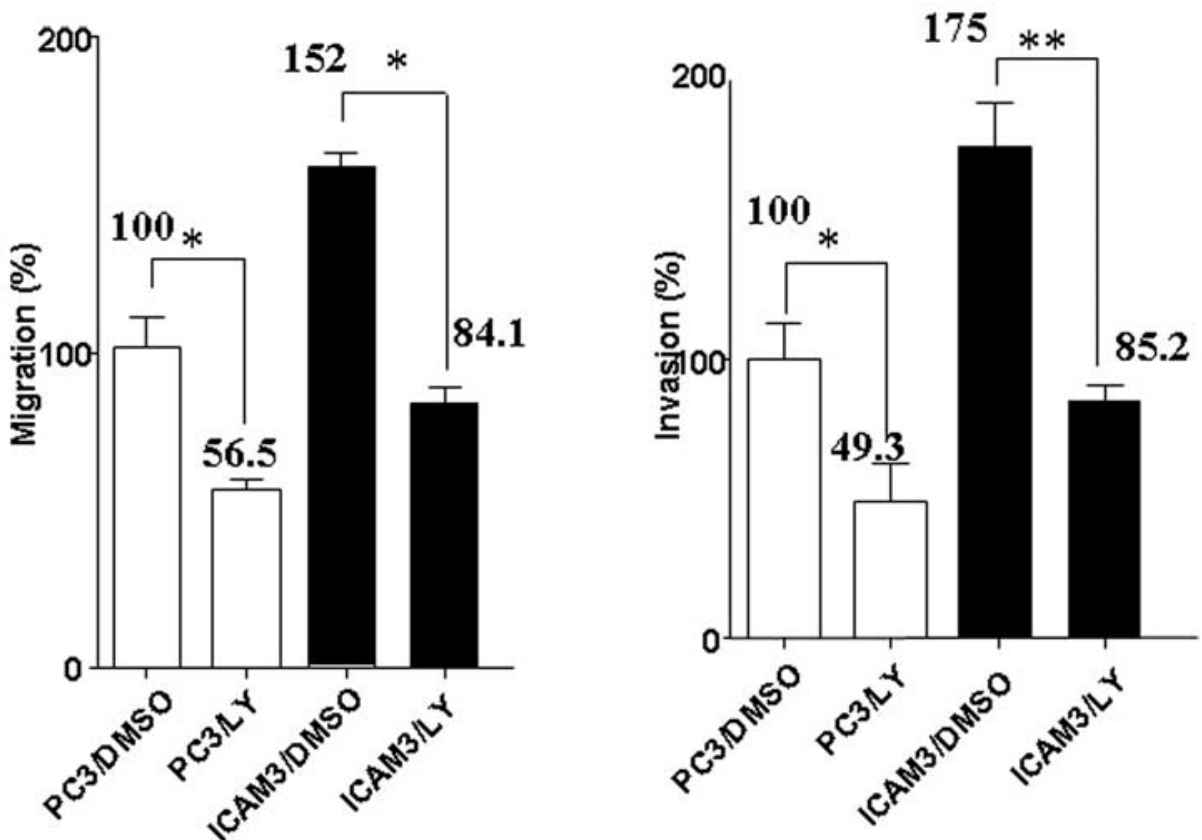

Figure 3. Akt is involved in ICAM-3-induced increase of cell migration and invasion. (A) Immunoblot assay of phospho-Akt or Akt was performed with NCIH1299 pcDNA3 or ICAM-3 stable transfectants. (B) Gelatin zymography. NCI-H1299 pcDNA3 or ICAM-3 stable transfectants were treated with DMSO or $20 \mu \mathrm{M}$ LY294002 for $18 \mathrm{~h}$ and conditional media were collected, and gelatin zymography was performed. LY represents LY294002-treated samples. (C and D) Migration assay (C) and invasion assay (D). NCI-H1299 pcDNA3 or ICAM-3 stable transfectants were treated with DMSO or $20 \mu \mathrm{M} \mathrm{LY} 294002$ for $18 \mathrm{~h}$. Treated cells were harvested and subjected to the migration or invasion assays. Mean values above each bar presented in (C) and (D) were calculated from three independent experiments and error bars indicate standard deviations (SD). p-values were calculated with use of the t-test. ${ }^{*} \mathrm{p}<0.05,{ }^{* *} \mathrm{p}<0.001$. 
A

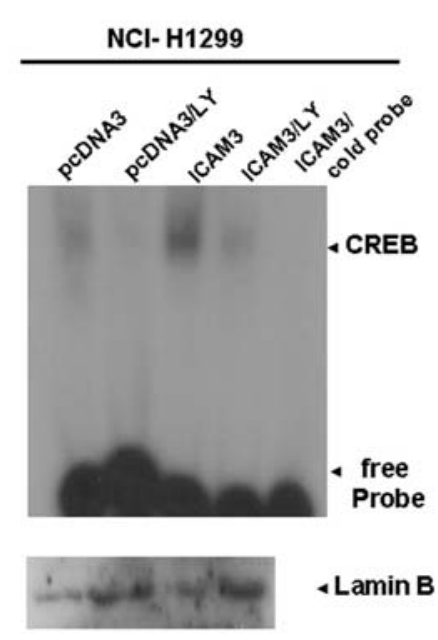

B
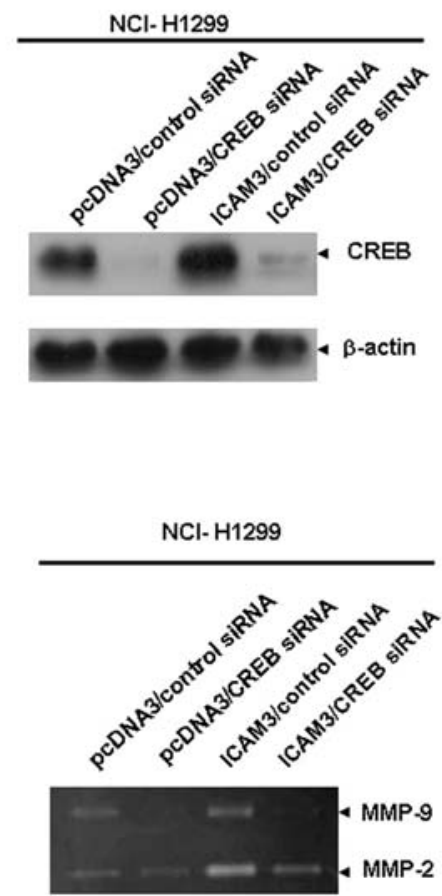

C

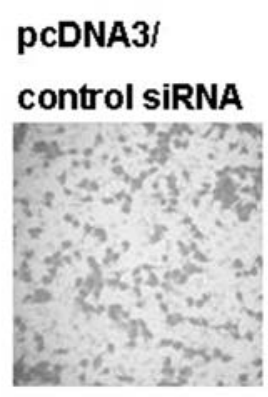

ICAM3!

control siRNA

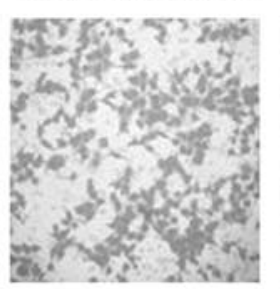

pcDNA3!

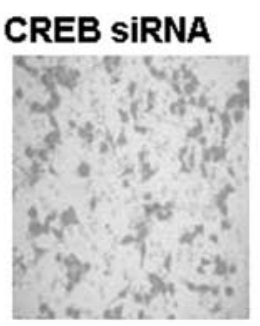

ICAM3!
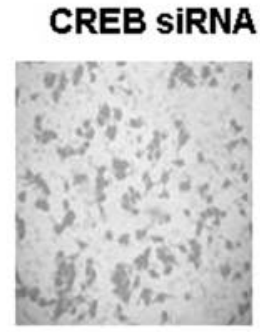

D

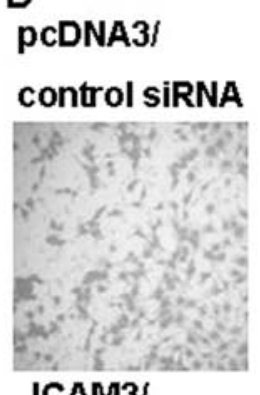

ICAM3/

pcDNA3!

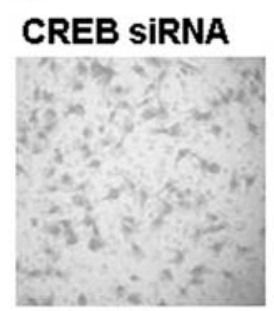

ICAM3!
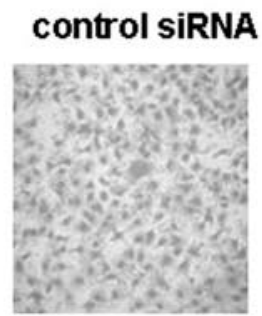
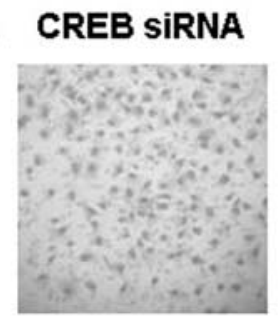

171.2
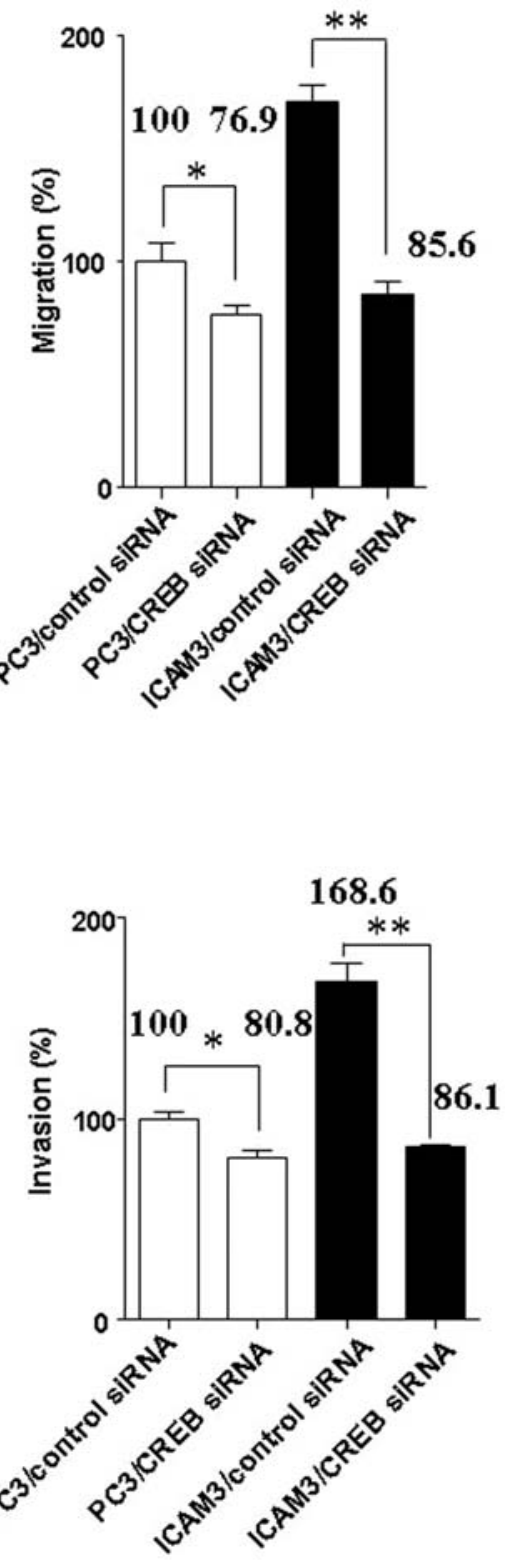

Figure 4. CREB activation through Akt is involved in ICAM-3-induced increase of cell migration and invasion. (A) EMSA assay determined that CREB is located at downstream of Akt in NCI-H1299 ICAM-3 stable transfectant. LY indicated LY294002-treated cells. NCI-H1299 pcDNA3 or ICAM-3 stable transfectants were treated with DMSO or $20 \mu \mathrm{M} \mathrm{LY} 294002$ for $18 \mathrm{~h}$, harvested, and an EMSA assay was performed. The lower panel indicated an immunoblot assay of lamin B. (B) Inhibition of CREB leads to downregulation of MMP-2 and MMP-9 activity. The upper panel showed the immunoblot assay of control siRNA- or CREB siRNA-treated NCI-H1299 pcDNA3 or ICAM-3 stable transfectants, while the lower panel depicts gelatin zymography of control siRNA-treated or CREB siRNA-treated NCI-H1299 pcDNA3 or ICAM-3 stable transfectants. (C and D) The decrease of CREB expression suppressed migration (C) and invasion (D). Data presented in (C) and (D) were derived from three independent experiments, and mean of three independent experiments were indicated. Error bars indicate standard deviations (SD) and p-values were calculated with the use of t-test. ${ }^{*} \mathrm{p}<0.05,{ }^{* *} \mathrm{p}<0.001$.

kinase/Akt pathway (24). The correlation between $\mathrm{p} 44 / 42$ MAPK activation and increased MMP-2 or MMP-9 levels was additionally investigated. In the immunoblot assay, increased p44/42 MAPK phosphorylation was detected, but PD98059, an inhibitor of p44/42 MAPK signaling pathway did not influence the activity of MMP-2 or MMP-9 (data not shown).

CREB is involved in ICAM-3-induced cell migration and invasion-intermediating signaling from Akt to MMP proteins.
Based on the previous reports that have indicated promotion of migration/invasion by Akt is related to the activity of various transcription factors $(11,29)$, we attempted to identify downstream targets of the Akt. An EMSA assay was performed to examine several transcription factor candidates, including AP-1, NF- $\mathrm{B}, \mathrm{SP}-1$, Oct-1 and CREB (data not shown). CREB alone was found to be in a constitutively activated state in NCI-H1299 ICAM-3 stable transfectant cells (Fig. 4A). Attenuated CREB activity after LY294002 treatment indicated that CREB is located downstream of the 
Akt (Fig. 4A). Immunoblot assay of lamin B was performed as a control for the nuclear fraction of EMSA assay (Fig. 4A, lower panel). To confirm the effects of CREB in the system, we treated stable transfectant cells with a specific CREB siRNA or control siRNA (Fig. 4B). Elimination of CREB by siRNA treatment was confirmed with immunoblot assay (Fig. 4B, upper panel). When activity of MMP proteins was examined by gelatin zymography, CREB siRNA-treated cells showed decreased MMP activity (Fig. 4B, lower panel). Migration and invasion assays were also performed, and CREB siRNA-treated ICAM-3 stable transfectants showed decreased migration and invasion by approximately $40-60 \%$ (Fig. 4C and D). These results indicated that CREB plays a role as a mediator between Akt and MMP, and ICAM-3/ $\mathrm{Akt} / \mathrm{CREB} / \mathrm{MMP}$ are present in the signaling pathway.

Transient transfection of ICAM-3 also induces increase of cell migration and invasion of human non-small cell lung cancer cells. Because NCI-H1299 pcDNA-3 or ICAM-3 stable transfectant clones only were used for the above results, we transfected with exogenous ICAM-3 to NCIH1299 cells transiently and performed cell migration and invasion assay (Fig. 5). As shown in Fig. 5, transiently increased ICAM-3 expression also enhanced cell migration and invasion in accordance with results of stable transfectants. To prove whether transiently increased ICAM-3 expression also induced the ICAM-3/Akt/CREB/MMP pathway, immunoblot assay was performed and specific pharmacological inhibitors (LY294002, OA-Hy and MMP-9 inhibitor I) were used. Expression of ICAM-3, phospho-Akt, phospho-CREB, MMP-2 and MMP-9 was increased (Fig. $5 \mathrm{Aa}$ ) and treatments of specific pharmacological inhibitors also suppressed cell migration (Fig. 5Ab) and invasion (Fig. 5Ac) in transiently increased ICAM-3 expressing cells. We also showed that elimination of CREB by specific siRNA treatment inhibited increase of cell migration and invasion by ICAM-3 in combination with CREB siRNA (Fig. 5B). In Fig. $5 \mathrm{~B}$, increase of ICAM-3 expression and decrease of CREB expression were confirmed by immunoblot assay (Fig. 5Ba). To confirm these results in NCI-H1299 cells, we transfected ICAM-3 into NCI-H460 NSCLC cells transiently, and then confirmed that increase of ICAM-3 expression in NCI-H460 cells also enhanced cell migration and invasion as shown in Fig. 6. These results indicated that the enhancement of cell migration and invasion by ICAM-3 in NCI-H460 cells was also dependent on the ICAM-3/Akt/CREB/MMP signaling pathway. This repeated the results obtained with NCI-H1299 cells, which was shown with immunoblot assay of phosphoAkt, phospho-CREB, MMP-2 and MMP-9 (Fig. 6Aa). Inhibition of Akt and MMP proteins by specific pharmacological inhibitors (LY294002, OA-Hy and MMP-9 inhibitor I) (Fig. 6Ab and Ac) and elimination of CREB by CREB siRNA treatments (Fig. 6Bb and $\mathrm{Bc}$ ) also decreased cell migration/invasion, which induced by ICAM-3 in NCI-H460 cells.

\section{Discussion}

Our present study suggests several new functions of ICAM-3. First, ICAM-3 induced increase in cancer cell migration/ invasion as confirmed by the wound-healing assay and migration/invasion assays using transwell plates (Fig. 1). We also found that these physiological changes resulted from elevated expression and activity of MMP-2 and -9 proteins, well-known inducers of cell migration and invasion (Fig. 2A and B). It was shown in several cancer types that various factors induced high expression MMP-2 and MMP-9; Ionizing radiation promote hepatocellular carcinoma cell invasion with increase of MMP-9 expression (4), enhancement of Bcl-2 expression increase gastric cancer cell invasion with increase of MMP-2 expression and activity (27), insulin-like growth factor-1 (IGF-1) induces invasion of distinct breast cancer cell lines (MDA-MB231, MDA-MB-435 and SUM159-PT) with induction of MMP proteins (30), and hepatitis $\mathrm{B}$ viral $\mathrm{HBx}$ protein increase hepatocellular carcinoma invasion potential with induction of MMP-9 gene expression (31). Our results also defined the role of ICAM-3 as an inducer of MMP proteins, and it increased cell migration and invasion via increase of activities of MMP-2 and -9. Treatments of specific pharmacological inhibitors to these MMP proteins suppressed cancer cell migration and invasion (32-34), which additionally indicates that ICAM-3 is an upstream effector to MMP proteins. Second, our results also have showed that enhancement of cell migration/invasion which was induced by ICAM-3 was composed of various components of intracellular signaling pathway. We found ICAM-3-Akt-CREB-MMPs pathway that intermediate migration and invasion induced by ICAM-3. As ICAM-3 activated Akt in our previous study (26), we postulated these proteins were involved in increased cell migration and invasion by ICAM-3. In our results, inhibition of Akt suppressed activity/expression of MMP and cell migration/ invasion, which agree with previous results of Bae et al, Weng et al and Yoeli-Lerner et al that PI3 kinase/Akt promotes cell migration and invasion through the induction of MMP proteins and modulation of MMP expression, and they also indicated that treatment of LY294002 suppressed cell migration and invasion (27,29,30). Du and Montminy reported that CREB is located at downstream of Akt, which induced phosphorylation of Ser-133 of CREB and then stimulated expression of the target gene via the activation of CREB (35). They also showed that Akt stimulated recruiting of CREB-binding protein (CBP) to CREB as well as phosphorylation of CREB. We also showed that elimination of CREB protein decreased activities of MMP proteins and then decreased cell migration/ invasion (Fig. 4B-D). It is known that CREB induces metastasis of melanoma via MMP-2 and the adhesion molecule such as MCAM/MUC18 (36), and dominant-negative mutant CREB has been shown to suppress metastasis (37). Therefore, our results suggest that ICAM-3-induced activity of MMP proteins and the increase of cell migration and invasion were intermediated by CREB. However, elimination of CREB protein failed to block migration and invasion perfectly, which imply that other signaling pathways or proteins might also affect migration and invasion in our system.

The enhancement of cell migration and invasion by ICAM-3 in NCI-H1299 cells did not show a relationship between ICAM-3, p53 and PTEN because NCI-H1299 cell line is $p 53$ - or PTEN-null, but NCI-H460 cell line contains 
A

a

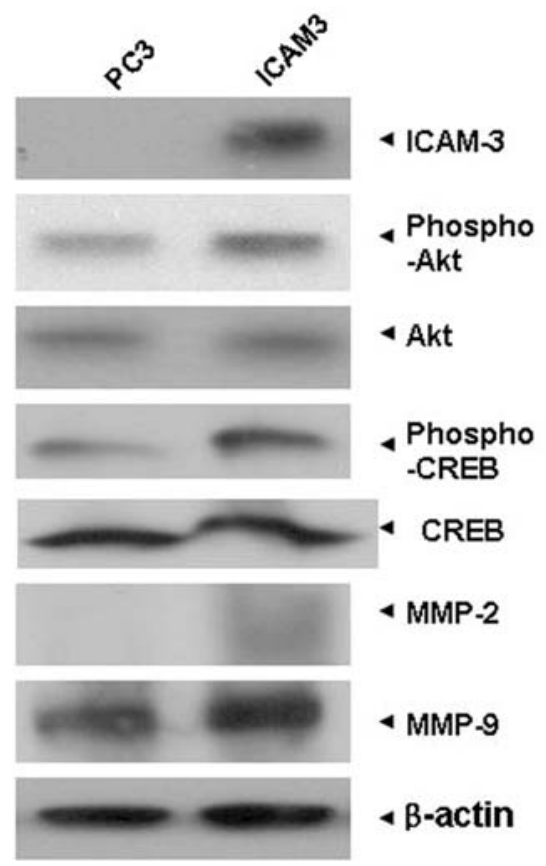

B

a
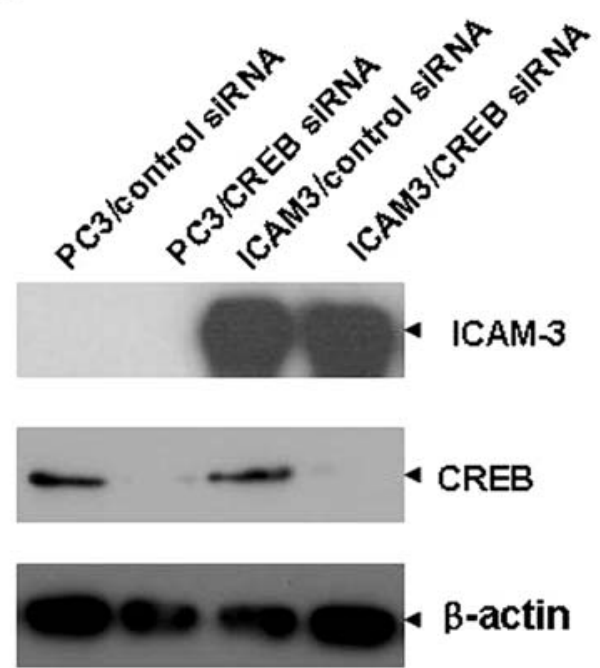

A

b

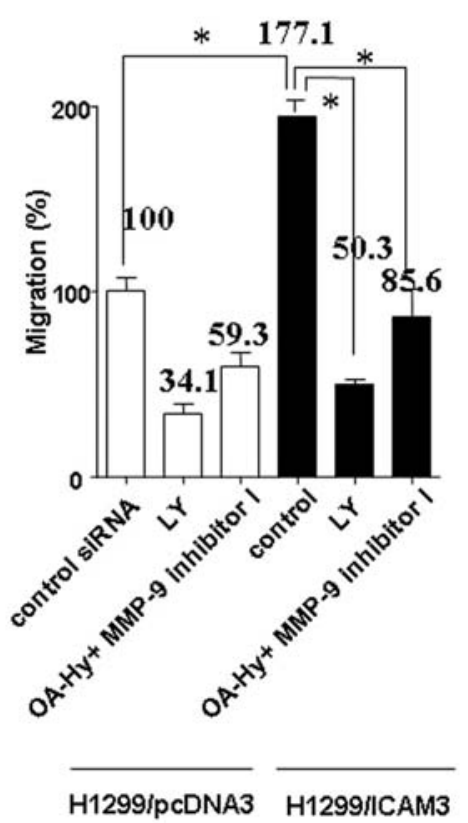

B

b

c
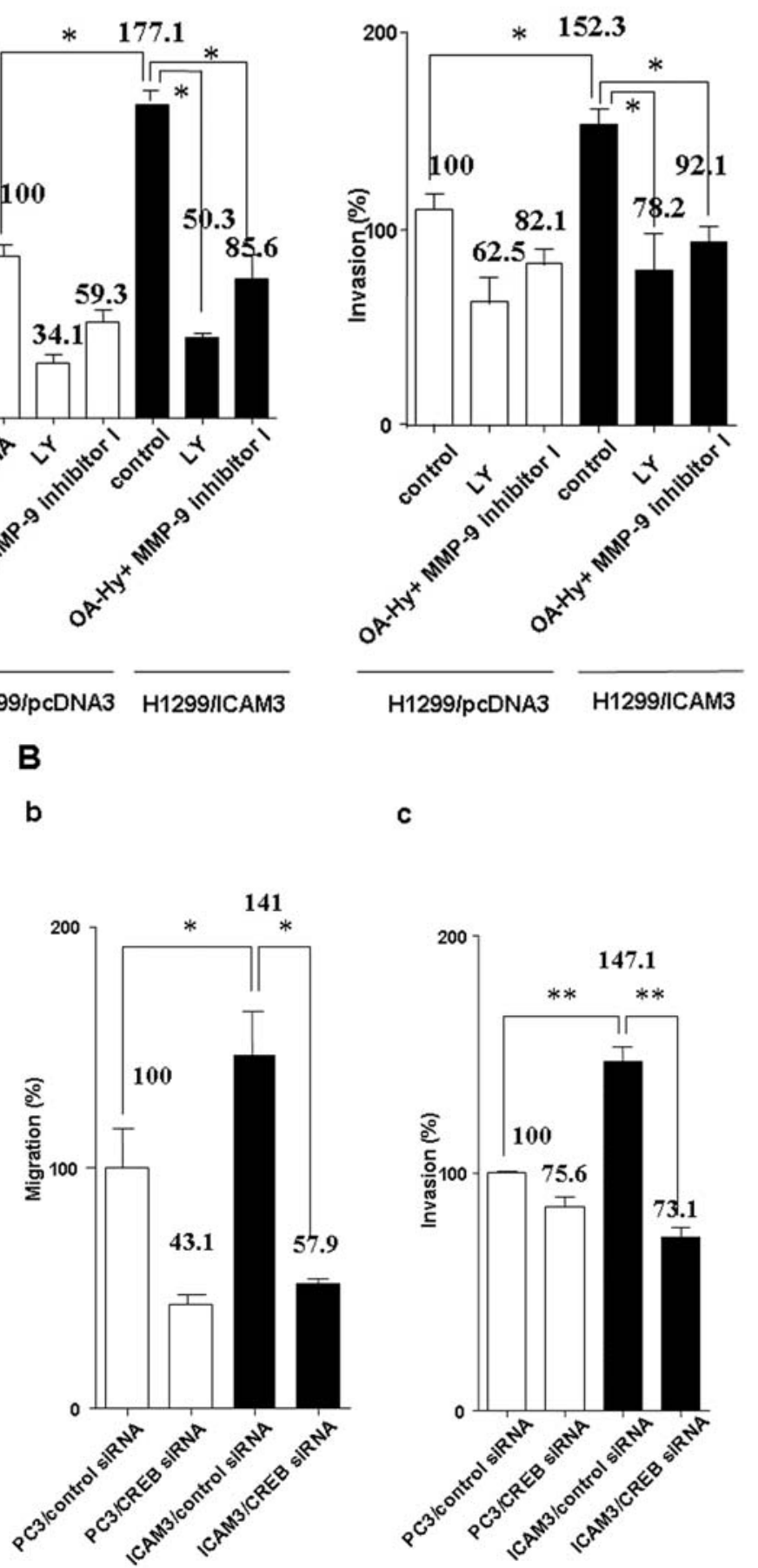

Figure 5. Transiently up-regulated ICAM-3 also increased cell migration/invasion of NCI-H1299. (A) NCI-H1299 cells were transiently transfected with pcDNA3 or ICAM-3, and immunoblot assay of ICAM-3, phospho-Akt, Akt, phospho-CREB, CREB, MMP-2 and MMP-9 were performed (Aa). ICAM-3transfected NCI-H1299 cells were pre-treated with DMSO, LY294002 or a combination of OA-Hy and MMP-9 inhibitor I for $18 \mathrm{~h}$, and then migration (Ab) and invasion assays (Ac) were performed as described in Materials and methods. LY indicated LY294002-treated cells. (B) NCI-H1299 cells also were cotransfected with combinations of pcDNA3 or ICAM-3 plasmids and control siRNA or CREB siRNA, and then and migration (Bb) and invasion assays (Bc) were performed. Efficiency of transfection was confirmed by immunoblot assay with ICAM-3 and CREB antibodies (Ba). Data presented were derived from three independent experiments, and each mean value of three independent experiments is indicated in the graphs. Error bars indicate standard deviations (SD) and $\mathrm{P}$ values were calculated with use of t-test. ${ }^{*} \mathrm{p}<0.05 ;{ }^{* *} \mathrm{p}<0.001$.

wild-type $p 53$ and PTEN (28). Several previous reports presented that both $\mathrm{p} 53$ and PTEN, major tumor suppressors, regulate cell migration and invasion (38-40); Moskovits et al and Gadea et al presented that p53 inhibits cell migration and invasion through suppression of chemokine stromal cellderived factor 1 (SDF-1/CXCL12) expression level and 
A

A

b

a

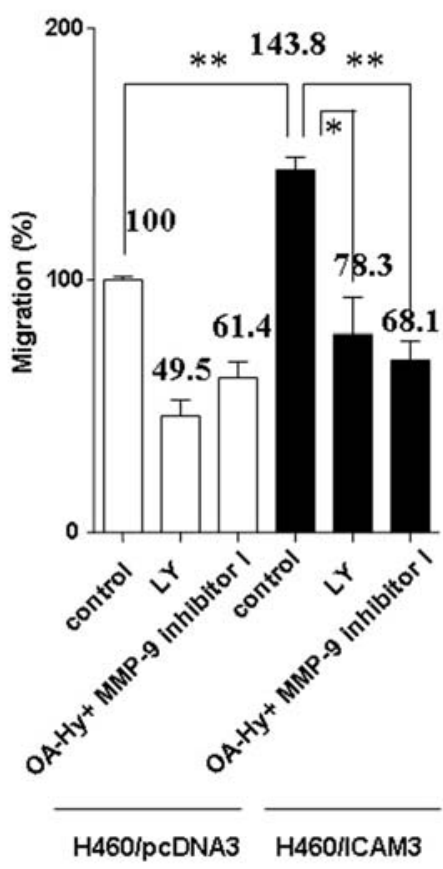

c

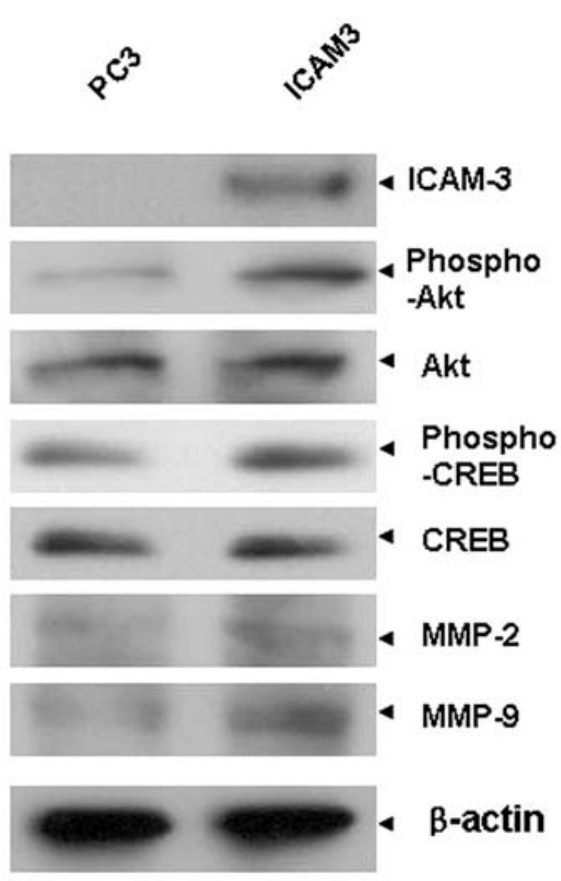

B

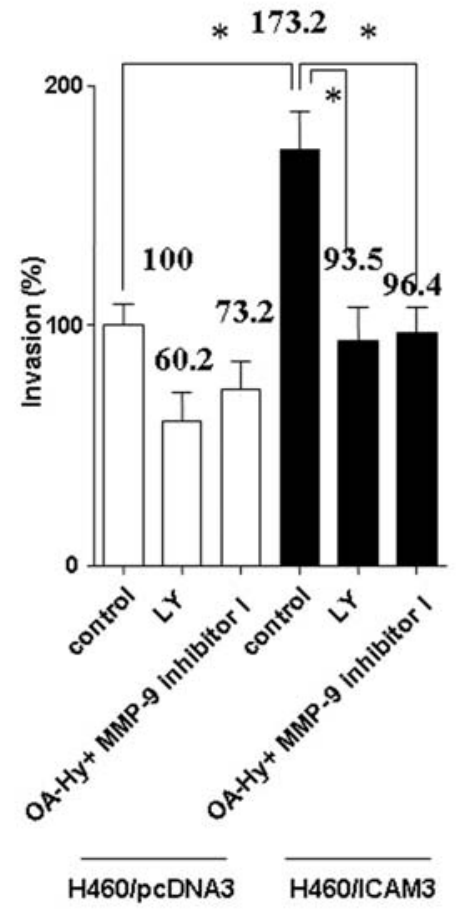

B

b

C

a
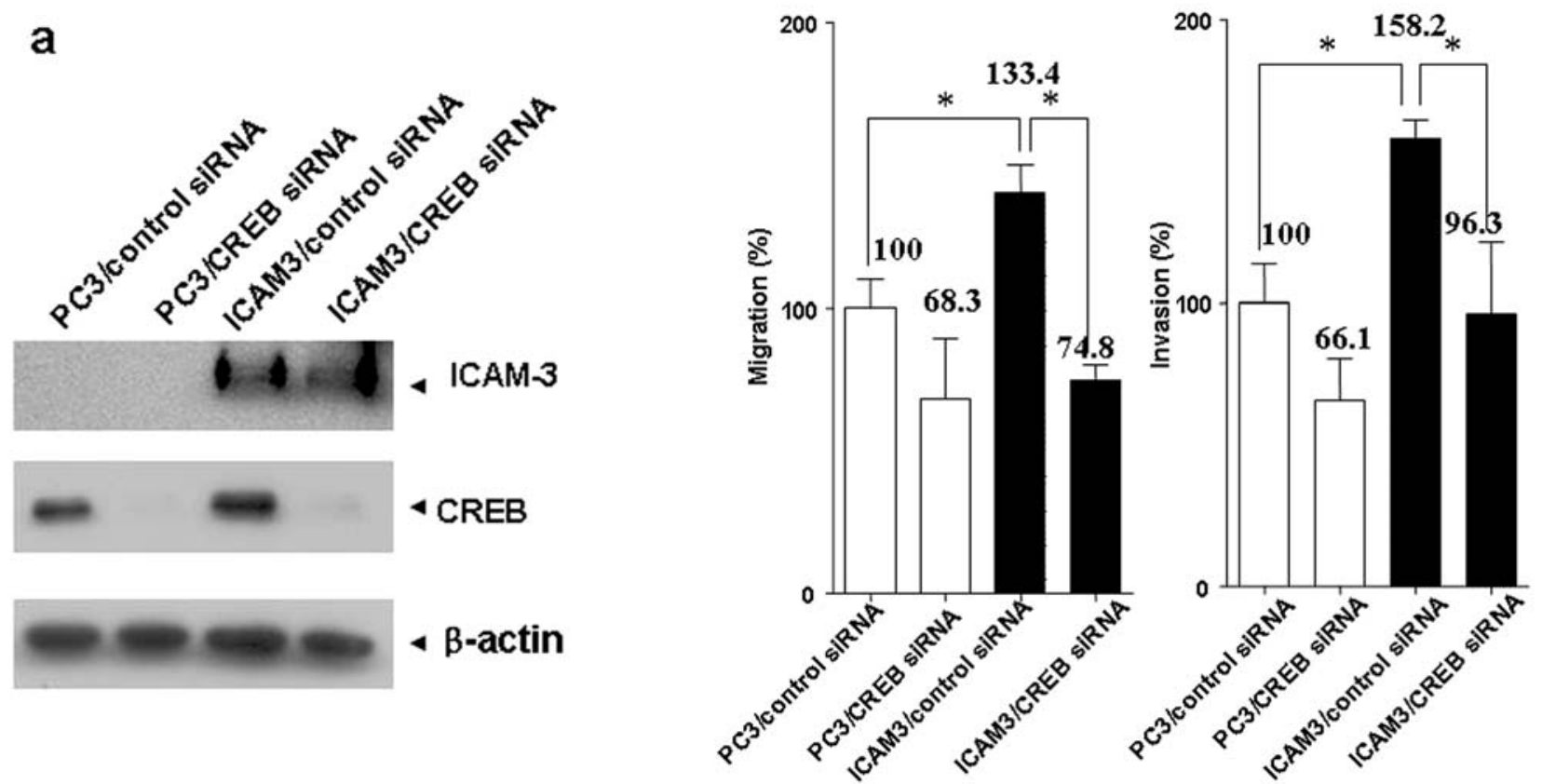

Figure 6. Transient transfection of ICAM-3 to NCI-H460 induced increased cell migration/invasion. (A) NCI-H460 cells were transiently transfected with pcDNA3 or ICAM-3, and then immunoblot assay of ICAM-3, phospho-Akt, Akt, phospho-CREB, CREB, MMP-2 and MMP-9 were performed (Aa). ICAM-3transfected NCI-H460 cells pre-treated with DMSO, LY294002 or a combination of OA-Hy and MMP-9 inhibitor I for $18 \mathrm{~h}$, and then migration (Ab) and invasion assays (Ac) were performed. LY indicates LY294002-treated cells. (B) NCI-H460 cells also were co-transfected with combinations of pcDNA3 or ICAM-3 plasmids and control siRNA or CREB siRNA, and immunoblot assay (Ba) was performed for detection of ICAM-3 and CREB. Migration (Bb) and invasion assays $(\mathrm{Bc})$ were performed with the co-transfected cells. Data presented were derived from three independent experiments, and mean values of three independent experiments were indicated. Error bars indicate standard deviations (SD) and P values were calculated with use of t-test. ${ }^{*} \mathrm{p}<0.05$, ${ }^{* *} \mathrm{p}<0.001$. 
RhoA-Rho-associated coil-containing protein kinase (ROCK) pathway, respectively $(38,39)$. Tamura et al reported that PTEN induces tyrosine dephosphorylation of FAK through direct interaction and inhibits cell migration and invasion (40). However, our results in Fig. 6 indicate that enhancement of cell migration and invasion by ICAM-3 were independent of intracellular p53 and PTEN status.

Taken together, our results suggest that ICAM-3 induced an increase in cell migration and invasion, and it was mediated via ICAM-3-Akt-CREB-MMP protein pathways, and these phenomena were not affected by $p 53$ and PTEN. In addition to increase of radio-resistance and cell proliferation, these results revealed a novel role of ICAM-3 in promoting migration and invasion. Furthermore, our present study supports the possibility that this signaling pathway may intermediate various cell phenotypes induced by ICAM-3. As Akt has a role in migration/invasion induced by ICAM-3, increase of migration and invasion in this study shared a common intracellular signaling pathway with promotion of cell proliferation in ICAM-3 over-expressing cells (26). Although we did not investigate the involvement of FAK in ICAM-3-induced cell migration and invasion in this study, increase of radiation resistance induced by ICAM-3 was dependent on FAK activation (25). Many investigators have reported that interaction of FAK with Akt have various roles in cellular adhesion, cell survival and drug resistance and radio-resistance (41-44). Therefore, we postulated that FAK could be one of the major components in ICAM-3-induced signaling.

Our results also suggest that ICAM-3 could be a therapeutic candidate for cancer treatment since ICAM-3 induced various intracellular signaling processes especially related to tumor malignancy and radio-resistance. Development of increased migration, invasion and radio-resistance in cancer patients presents very serious obstacles for cancer treatment. However, few studies have described the intracellular mechanism underlying the relationship between migration/invasion and radio-resistance, although radiation is known as one of the extracellular stresses that affects cancer invasion, which involves induction of expression of MMP proteins and modification of the cytoskeleton $(45,46)$. A more comprehensive study, especially with the use of in vivo systems, on effect of ICAM-3 signaling and related mechanisms is required to understand the role of ICAM-3 in cancer, and a series of in vivo studies may provide evidence that would facilitate the treatment of cancer. To date, the use of monoclonal antibodies, antisense oligonucleotides and small molecules abolishing the action ICAM-1 have been generated for treatment of anti-inflammation and rheumatoid arthritis (47). Our findings on ICAM-3 and the associated signaling pathways may suggest new therapeutic targets to treat immune diseases and cancer.

\section{Acknowledgements}

This work was supported by Nuclear Research \& Development Program (M20702020002-08N0202-00210) and in part by Basic Science Research Program (R11-2008-014-01001-0) of the Korea Science and Engineering Foundation (KOSEF) grant funded by the Korean government (MEST) (H.-D. Um).

\section{References}

1. Friedl $\mathrm{P}$ and Wolf $\mathrm{K}$ : Tumour-cell invasion and migration: diversity and escape mechanisms. Nat Rev Cancer 3: 362-374, 2003.

2. Weber GF: Molecular mechanisms of metastasis. Cancer Lett 270: 181-190, 2008.

3. Gupta GP and Massague J: Cancer metastasis: building a framework. Cell 127: 679-695, 2006.

4. Cheng JC, Chou CH, Kuo ML and Hsieh CY: Radiationenhanced hepatocellular carcinoma cell invasion with MMP-9 expression through PI3-Kinase/Akt/NF-kB signal transduction pathway. Oncogene 25: 7009-7018, 2006.

5. Chen PN, Hsieh YS, Chiou HL and Chu SC: Silibinin inhibits cell invasion through inactivation of both PI3-Kinase/Akt and MAPK signalling pathways. Chem Biol Interact 156: 141-150, 2005.

6. Kaiser U, Auerbach B and Oldenburg M: The neural cell adhesion molecule NCAM in multiple myeloma. Leuk Lymphoma 20: 389-395, 1996.

7. Watine J and Friedberg B: Laboratory variables and stratification of metastatic colorectal cancer patients: recommendations for therapeutic trials and for clinical practice guidelines. Clin Chim Acta 345: 1-15, 2004.

8. Simmons D, Makgoba MW and Seed B: ICAM, an adhesion ligand of LFA-1, is homologous to the neural cell adhesion molecule NCAM. Nature 331: 624-627, 1998.

9. Springer TA: Traffic signals for lymphocyte recirculation and leukocyte emigration: the multistep paradigm. Cell 76: 301-314, 1994.

10. Hayflick JS, Kilgannon P and Gallatin WM: The intercellular adhesion molecule (ICAM) family of proteins. New members and novel functions. Immunol Res 17: 313-327, 1998.

11. Toivanen A, Ihanus E, Mattila M, Lutz HU and Gahmberg CG: Importance of molecular studies on major blood groupsintercellular adhesion molecule-4, a blood group antigen involved in multiple cellular interactions. Biochim Biophys Acta 1780: 456-466, 2008.

12. Gahmberg CG, Tian L, Ning L and Nyman-Huttunen H: ICAM-5 - a novel two-facetted adhesion molecule in the mammalian brain. Immunol Lett 117: 131-135, 2008.

13. Figdor CG, van Kooyk Y and Adema GJ: C-type lectin receptors on dendritic cells and Langerhans cells. Nat Rev Immunol 2: 77-84, 2002

14. Geijtenbeek TBH, Krooshoop DJEB, Bleijs DA, et al: DCSIGN-ICAM-2 interaction mediates dendritic cell trafficking. Nat Immunol 1: 353-357, 2000

15. Maeda K, Kang SM, Sawada T, et al: Expression of intercellular adhesion molecule-1 and prognosis in colorectal cancer. Oncol Rep 9: 511-514, 2002.

16. Tachimori A, Yamada N, Sakate Y, et al: Up regulation of ICAM-1 gene expression inhibits tumour growth and liver metastasis in colorectal carcinoma. Eur J Cancer 41: 1802-1810, 2005.

17. Johnson JP: Cell adhesion molecules in the development and progression of malignant melanoma. Cancer Metastasis Rev 18: 345-357, 1999.

18. Kobayashi H, Boelte KC and Lin PC: Endothelial cell adhesion molecules and cancer progression. Curr Med Chem 14: 377-386, 2007.

19. Natali PG, Hamby CV, Felding-Habermann B, et al: Clinical significance of alpha(v)beta3 integrin and intercellular adhesion molecule-1 expression in cutaneous malignant melanoma lesions. Cancer Res 57: 1554-1560, 1997.

20. Huang WC, Chan ST, Yang TL, Tzeng CC and Chen CC: Inhibition of ICAM-1 gene expression, monocyte adhesion and cancer cell invasion by targeting IKK complex: molecular and functional study of novel alpha-methylene-gamma-butyrolactone derivatives. Carcinogenesis 25: 1925-1934, 2004.

21. Rosette C, Roth RB, Oeth P, et al: Role of ICAM1 in invasion of human breast cancer cells. Carcinogenesis 26: 943-950, 2005.

22. Montazeri A, Kanitakis J, Zebrine G, Bourchany D, Schmitt D and Claudy A: Expression of ICAM-3/CD50 in normal and diseased skin. Br J Dermatol 133: 377-384, 1995.

23. Molica S, Dattilo A, Mannella A and Levato D: Intercellular adhesion molecules (ICAMs) 2 and 3 are frequently expressed in B cell chronic lymphocytic leukemia. Leukemia 10: 907-908, 1996.

24. Mousa SA: Cell adhesion molecules: potential therapeutic \& diagnostic implications. Mol Biotechnol 38: 33-40, 2008. 
25. Chung YM, Kim BG, Park CS, et al: Increased expression of ICAM-3 is associated with radiation resistance in cervical cancer. Int J Cancer 117: 194-201, 2005.

26. Kim YG, Kim MJ, Lim JS, Lee MS, Kim JS and Yoo YD: ICAM-3-induced cancer cell proliferation through the PI3K/Akt pathway. Cancer Lett 239: 103-110, 2006.

27. Bae IH, Park MJ, Yoon SH, et al: Bcl-w promotes gastric cancer cell invasion by inducing matrix metalloproteinase-2 expression via phosphoinositide 3-kinase, Akt, and Sp1. Cancer Res 66: 4991-4995, 2006.

28. Park JK, Jung HY, Park SH, et al: Combination of PTEN and gamma-ionizing radiation enhances cell death and $\mathrm{G}(2) / \mathrm{M}$ arrest through regulation of AKT activity and p21 induction in nonsmall-cell lung cancer cells. Int J Radiat Oncol Biol Phys 70: 1552-1560, 2008.

29. Weng CJ, Chau CF, Hsieh YS, Yang SF and Yen GC: Lucidenic acid inhibits PMA-induced invasion of human hepatoma cells through inactivating MAPK/ERK signal transduction pathway and reducing binding activities of NF-kappaB and AP-1. Carcinogenesis 29: 147-156, 2008.

30. Yoeli-Lerner $\mathrm{M}$ and Toker A: Akt/PKB signaling in cancer: a function in cell motility and invasion. Cell Cycle 5: 603-605, 2006.

31. Chung TW, Lee YC and Kim CH: Hepatitis B viral HBx induces matrix metalloproteinase-9 gene expression through activation of ERK and PI-3K/AKT pathways: involvement of invasive potential. FASEB J 18: 1123-1125, 2004.

32. Kargozaran H, Yuan SY, Breslin JW, et al: A role for endothelial-derived matrix metalloproteinase- 2 in breast cancer cell transmigration across the endothelial-basement membrane barrier. Clin Exp Metastasis 24: 495-502, 2007.

33. Levin JI, Chen J, Du M, et al: The discovery of anthranilic acidbased MMP inhibitors. Part 2: SAR of the 5-position and P1(1) groups. Bioorg Med Chem Lett 11: 2189-2192, 2001.

34. Carnero A, Blanco-Aparicio C, Renner O, Link W and Leal JF: The PTEN/PI3K/AKT signalling pathway in cancer, therapeutic implications. Curr Cancer Drug Targets 8: 187-198, 2008.

35. Du K and Montminy M: CREB is a regulatory target for the protein kinase Akt/PKB. J Biol Chem 273: 32377-32379, 1998.

36. Jean D and Bar-Eli M: Regulation of tumor growth and metastasis of human melanoma by the CREB transcription factor family. Mol Cell Biochem 212: 19-28, 2000.

37. Aucoin R, Reiland J, Roy M and Marchetti D: Dominantnegative CREB inhibits heparanase functionality and melanoma cell invasion. J Cell Biochem 93: 215-223, 2004.
38. Moskovits N, Kalinkovich A, Bar J, Lapidot T and Oren M: p53 attenuates cancer cell migration and invasion through repression of SDF-1/CXCL12 expression in stromal fibroblasts. Cancer Res 66: 10671-10676, 2006.

39. Gadea G, de Toledo M, Anguille C and Roux P: Loss of p53 promotes RhoA-ROCK-dependent cell migration and invasion in 3D matrices. J Cell Biol 178: 23-30, 2007.

40. Tamura M, Gu J, Takino T and Yamada KM: Tumor suppressor PTEN inhibition of cell invasion, migration, and growth: differential involvement of focal adhesion kinase and p130Cas. Cancer Res 59: 442-449, 1999.

41. Basson MD: An intracellular signal pathway that regulates cancer cell adhesion in response to extracellular forces. Cancer Res 68: 2-4, 2008.

42. Xia H, Nho RS, Kahm J, Kleidon J and Henke CA: Focal adhesion kinase is upstream of phosphatidylinositol 3-kinase/ Akt in regulating fibroblast survival in response to contraction of type I collagen matrices via a beta 1 integrin viability signaling pathway. J Biol Chem 279: 33024-33034, 2004.

43. Fraser M, Leung B, Jahani-Asl A, Yan X, Thompson WE and Tsang BK: Chemoresistance in human ovarian cancer: the role of apoptotic regulators. Reprod Biol Endocrinol 1: 66, 2003.

44. Carlson M, Longaker J and Thompson JS: Modulation of FAK, Akt, and p53 by stress release of the fibroblast-populated collagen matrix 1, 2. J Surg Res 120: 171-177, 2004.

45. Park C-M, Park M-J, Kwak H-J, et al: Ionizing radiation enhances matrix metalloproteinase-2 secretion and invasion of glioma cells through Src/epidermal growth factor receptormediated p38/Akt and phosphatidylinositol 3-kinase/Akt signaling pathways. Cancer Res 66: 8511-8519, 2006.

46. Beinke C, van Beuningen D and Cordes N: Ionizing radiation modulates expression and tyrosine phosphorylation of the focal adhesion-associated proteins focal adhesion kinase (FAK) and its substrates p130cas and paxillin in A549 human lung carcinoma cells in vitro. Int J Radiat Biol Phys 79: 721-731, 2003.

47. Hideshima T, Chauhan D, Podar K, Schlossman RL, Richardson P and Anderson KC: Novel therapies targeting the myeloma cell and its bone marrow microenvironment. Semin Oncol 28: 607-612, 2001. 